\title{
Severe anaemia and multiple ecchymoses in a postpartum female due to acquired haemophilia $A$
}

\author{
Palangasinghe DR ${ }^{1}$, Gamage VNU' ${ }^{1}$, Bodinayake CK${ }^{1}$, Mohotti MGM ${ }^{2}$ \\ ${ }^{\prime}$ University Medical Unit, Teaching Hospital, Karapitiya, Galle, Sri Lanka. \\ ${ }^{2}$ Haematology Unit, Teaching Hospital, Karapitiya, Galle, Sri Lanka.
}

Correspondence: Dr. Dhammika Palangasinghe

e-mail:dhammika27@yahoo.com

\section{Introduction}

Acquired haemophilia A (AHA) is a rare acquired bleeding disorder with a worldwide incidence of 2 per million of the population (1). It has a bimodal age distribution including postpartum period and adults over 50 years while the latter being most frequent (2). We report a case of AHA in a postpartum young female. We were unable to trace previous cases of the same condition reported in Sri Lanka.

\section{Case Report}

A 26-year old previously healthy lactating mother presented at postpartum $8^{\text {th }}$ week with gradual onset left hip joint pain for 2 weeks duration.

Four weeks prior to this presentation she had developed multiple bruises over lower limbs and a painful swelling of the left calf which resolved spontaneously. She denied any pain involving other joints while left hip pain was progressive leading to difficulty in walking.

New bruises had appeared over forearms and thighs with an intermittent low grade fever for one week prior to admission.

There was no history of easy bruising or any bleeding disorder in her past or in the family. No excessive bleeding occurred following trauma or surgery in the past.

On examination she was pale, anicteric, afebrile with multiple ecchymoses and bruises over forearms and thighs. She was keeping left lower limb flexed at hip joint demonstrating psoas muscle irritation. Abdomen was soft and non-tender with well healed LSCS scar. No hepatosplenomegaly or pelvic masses were felt. Examination of cardiovascular, respiratory and nervous system was unremarkable.
On admission, haemoglobin was $5.9 \mathrm{~g} / \mathrm{dL}$, white cells $12,000 / \mathrm{mm}^{3}$ and platelets $631,000 / \mathrm{mm}^{3}$. Blood picture revealed severe normochromic normocytic anaemia with evidence of acute bleeding. Reticulocyte count was $7.8 \%$. Prothrombin time was 11.4 seconds (control $11.4 \mathrm{sec}$ ). Activated Partial Thromboplastin Time (APTT) was 96 seconds (control $44 \mathrm{sec}$ ). Bleeding time was normal (6 minutes).

APTT correction studies and inhibitor screening were performed.

APTT after $50: 50$ mixing with normal plasma: 57 $\sec$ (corrected)

After 2 hour incubation at $37^{\circ} \mathrm{C}$,

APTT of pre mixed sample : $85 \mathrm{sec}$

APTT of fresh mixed sample : $66 \mathrm{sec}$

Results were concluded as presence of time and temperature dependent coagulation inhibitor and AHA was diagnosed by the characteristic time and temperature dependent nature of factor VIII (FVIII) inhibitors.

Ultrasound scan (USS) revealed a large haematoma in the left psoas muscle $(24 \mathrm{~cm} \times 5 \mathrm{~cm} \times 3 \mathrm{~cm})$. Liver, renal functions and UFR were normal. CRP 112 $\mathrm{mg} / \mathrm{dL}$, ESR $120 \mathrm{~mm}$ in the $1^{\text {st }}$ hour while all the cultures were sterile. ANA, Rheumatoid factor were negative. Complement levels were within normal limits.

Soon after the diagnosis she was started on oral prednisolone $1 \mathrm{mg} / \mathrm{kg} /$ day in order to eradicate the inhibitor. She required recombinant factor VIIa $90 \mu \mathrm{g} / \mathrm{kg}$ two doses for the prevention of further bleeding. Supportive care was given with Red cell transfusion $(1,500 \mathrm{~mL})$, tranexamic acid, iron and folate. Response was assessed clinically, 
ultrasonographically and haematologically(Table 1). She achieved a remarkable clinical response by $4^{\text {th }}$ day of therapy with US showing evidence of resolving haematoma.

\section{AHA are rarely reported (6).}

Patients require close followup for the initial 6 months and thorough haemostatic assessment prior to any invasive procedure.

Table 1: Progress of APTT \& Ab

\begin{tabular}{lllllll}
\hline Day of steroid therapy & Day $\mathbf{1}$ & Day 2 & Day 3 & Day 4 & Day $\mathbf{5}$ & Day 10 \\
\hline APTT $(\mathrm{sec})$ & 96 & 87 & 68 & 57 & 48 & 35 \\
Haemoglobin $(\mathrm{g} / \mathrm{dl})$ & 5.9 & 9.6 & 9.6 & 10.0 & 10.3 & 11.2 \\
\hline
\end{tabular}

Prednisolone was continued in same dose for 3 weeks and tapered. No recurrences were detected and the patient remained asymptomatic at the end of 3 months after discharge.

\section{Discussion}

AHA is a rare potentially life threatening disease caused by the development of autoantibodies against FVIII protein resulting in impaired function of FVIII. It is associated with systemic autoimmune disorders, haematological malignancies, drugs, pregnancy, postpartum and sometimes idiopathic (3).

Bleeding pattern is distinct from congenital haemophilia with mucocutaneous and muscle bleeding in contrast to haemarthrosis.

AHA should be suspected in the presence of recent onset bleeding in an adult together with isolated prolongation of APTT and normal bleeding time. Further, mixing studies including $2 \mathrm{~h}$ incubation at $37^{\circ} \mathrm{C}$ are necessary to identify time and temperature dependent FVIII inhibitors.

Goals of therapy are inhibitor eradication and prevention of further bleeding. Corticosteroids or/and cyclophosphamide are recommended as the first line treatment while rituximab with immune suppressives are used as the second line. By passing agents are used to control bleeding. Activated Prothombin Complex Concentrates and recombinant FVIIa have equal efficacy (4). Our patient was treated with the latter as it was freely available to us. High dose FVIII combined with plasmapheresis and immunoadsorption is considered in refractory cases (4).

Postpartum AHA is very rare but carries a good prognosis (5). There is a possibility of recurring in subsequent pregnancies and cases with recurrent
Even though it may be challenging, this case report highlights the importance of high degree of clinical suspicion for early diagnosis and prompt treatment of AHA in order to prevent life threatening outcomes.

\section{Conclusions}

Although rare, AHA could present with life threatening bleeding during postpartum period. Prompt recognition and treatment is necessary to prevent adverse outcomes.

\section{References}

1. P. Giangrande. Acquired Hemophilia WFH.ORG November 2012; 1186.

2. Lan Mo and George C Bao. Acquired factor VIII deficiency: two case reports and a review of literature. Exp Hematol Oncol. 2017; 6-8.

3. Sara J Grethlein, Craig M Kessler. Acquired Haemophilia: Practice essentials, Background, Pathophysiology. emedicine.medscape.com/article/211186.

4. Peter W Collins. Diagnosis and management of acquired coagulation inhibitors: a guideline from UKHCDO. British Journal of Haematology 2013 September; 162: 758-73.

5. Prognostic factors for remission of and survival in acquired hemophilia A: results from the GTH-AH 01/2010 study. Blood 2015; 125: 1091-7.

6. Mulleiz SM, Vantilburgh A, Devreese KM. Acquired Hemophilia: a case report and review of the literature. Int JLab Hematol 2014 Jun; 36(3): 398-407. 